\title{
Numerical simulation of European option payoff based on stochastic differential delay equations
}

\author{
Hui Yu
}

College of Science, Heilongjiang Bayi Agricultural University, Daqing 163319, China

Corresponding Author Email: yuhui163@163.com

https://doi.org/10.18280/mmep.050207

Received: 22 May 2018

Accepted: 25 June 2018

\section{Keywords:}

stochastic differential delay equations, European option payoff, Euler-Maruyama method, Monte Carlo method

\begin{abstract}
The purpose of the paper is to conduct the numerical simulation for the payoff of European option based on stochastic differential delay equations, and three results are given. Firstly, by means of simulating the delay factor, the classical Black-Scholes financial model is extended to the generalized Black-Scholes model, which is described by stochastic differential delay equations (SDDEs). Secondly, in view of the hardship that it is difficult to obtain the exact solutions of SDDEs, the algorithm, which includes the Euler-Maruyama method and Monte Carlo method, is introduced to such generalized model of SDDEs. Via controlling the global error of the final time asset price for determining the step-size and the number of sample path, the algorithm is given. Thirdly, the mean-square error of the algorithm is analysed in the simulation of European option payoff. Finally, the related numerical experiments are conducted according to the main conclusions. The obtained results may serve as the simulation of European option payoff.
\end{abstract}

\section{INTRODUCTION}

In some ways, the world is full of randomness. With the efforts of many scientists of several centuries, in 1949, the Japanese scientist Ito established the theory of stochastic differential equations (SDEs) and developed the qualitative and quantitative analysis methods for random phenomenon. In recent decades, the SDEs are important and applied widely in many fields such as medicine, economics, physics, biology, and control science [1-3].

In the financial market and other areas, it is meaningful and significant to model the impact of the stochastic factors ${ }^{[4,5]}$. The classical Black-Scholes model in financial markets was first studied by means of SDEs [6]. With the development of mathematics theory, the classical Black-Scholes model of SDEs was extended from different angles. In previous studies $[8,9]$, the classical model was improved by broadening the Hurst parameter from $\mathrm{H}=1 / 2$ to $H \in(0,1)$, which can capture the characteristic of long-range dependence and heavy tailed distribution in miscellaneous financial data. In other studies ${ }^{[10]}$, the model was revised by introducing $\theta$ process. However, the exact solutions of SDDEs can hardly be obtained. Hence, investigating appropriate numerical methods and studying their properties, which can be classified into strong and weak approximations [11-16], are very important both in theory and in application.

But now, the results about European option payoff described by SDDEs was very few. The major aim here is to fill this gap in numerical simulation.

\section{PRELIMINARY STEPS}

This chapter studies Ito's SDDEs model in financial mathematics.

$$
\begin{aligned}
& d S(t)=\gamma S(t) \mathrm{d} t+V(S(t-\tau)) S(t) \mathrm{d} W(t) \quad 0 \leq t \leq T \quad \tau \in \mathrm{R}_{+} \\
& S(t)=\xi(t),-\tau \leq t \leq 0, \xi(t) \in C\left([-\tau, 0] ; R_{+}\right) \\
& T=m \tau \quad m \in \mathrm{R}_{+}
\end{aligned}
$$

Assuming the equation is one-dimensional, $S(t)$ represents the market price of an asset at time $t ; \tau>0$ is time lag; $\gamma>0$ stands for risk interest rate; $V$ is perturbation function; $W(t)$ is a one-dimensional Brown movement; $T$ is the final moment. The purpose is to simulate the profitability of European options and to discuss the second-moment error of simulated profits.

Assume 1. Assuming that the wave function $V \in\left(R_{+} ; R_{+}\right)$ satisfies global bounded and local Lipschitz conditions, that is:

$V(x) \leq K, \forall x \geq 0, V(x)=V(0), x<0$

For every $R>0$, the existing $\mathrm{K}_{\mathrm{R}}>0$ makes:

$|V(x)-V(\bar{x})| \leq K_{R}|x=\bar{x}|, \forall x, \bar{x} \in[0, R]$

Assume 2. Assuming that $\xi$ satisfies the Holder continuity of $\gamma \in(0,1 / 2]$ that is:

$$
\sup _{-\tau \leq u<v \leq 0} \frac{|\xi(v)-\xi(u)|}{(v-u)^{\gamma}}<\infty
$$

The Model (1) discussed in this chapter is the special case of the autonomous delay differential equation which satisfies Assumptions 1 and 2. This model has a unique positive solution or non-negative solution. Since the solution has a finite probability expectation, a variety of options-related quantities can be established; 
The $\operatorname{Eq}(1)$ is insensitive to small changes in the time delay $\tau$, so the asset price $S(t)$ and its associated option price do not change much; the various quantities associated with the option can be simulated numerically, not just theoretically.

In this chapter, the advantages of applying Euler-Maruyama and Monte Carlo algorithms are that the choice of step size can reflect the global error of the asset price simulation at the final moment. In addition, the conditions for option profitability studied in this chapter are few. The numerical simulation method also has weaker requirements for the conditions of option profit. This is more general, so the application scope is relatively wide.

Regarding the numerical method of $\operatorname{Eq}(1)$, many literatures have been studied. If $h=\tau / M, \mathrm{M} \in N_{+}$, then the Euler-Maruyama method has a strong convergence order of $1 / 2$ order and a weak convergence order of 1 order. This section introduces the concept of strong convergence order and weak convergence order. When the problem of orbit simulation for a stochastic system is required, a strongly convergent discrete format must be constructed so that the numerical solution converges to the analytical solution in a mean-square or almost-ambiguous sense. Sometimes, for a stochastic system, it is not the shape of the orbit, but a statistic of it, such as the first moment, the second moment, etc. The numerical solution given by the discretization scheme does not have to converge strongly, but only needs to satisfy the weak convergence.

Definition 1 Divide the time interval $[0, T]$ : $0=\mathrm{t}_{0}<\mathrm{t}_{1}<\ldots<\mathrm{t}_{\mathrm{N}}=\mathrm{T}$, and record the corresponding numerical solution that is $\bar{X}_{i}, i=0, \ldots, N$, then the strict definition of $\gamma$ squared mean convergence is as follows:

$$
\mathrm{E}\left(\left\|\bar{X}_{N}-X_{T}\right\|^{2}\right) \leq C \Delta^{2 \gamma}, \Delta=\max _{0 \leq i \leq N-1}\left(t_{i+1}-t_{i}\right)
$$

$\mathrm{C}$ is a constant that is independent of $\Delta$, and $\gamma$ is an integer or half-integer.

Definition 2 Divide the time interval $[0, \mathrm{~T}]$ : $0=\mathrm{t}_{0}<\mathrm{t}_{1}<\ldots<\mathrm{t}_{\mathrm{N}}=\mathrm{T}$, and record the corresponding numerical solution that is $\bar{X}_{i}, i=0, \ldots, N g$ is a function, so the strict definition of $\gamma$-order weak convergence is as follows:

$\left|\mathrm{E}\left[\mathrm{g}\left(\bar{X}_{N}\right)-g\left(X_{T}\right)\right]\right| \leq C \Delta^{\gamma}, \Delta=\max _{0 \leq i \leq N-1}\left(t_{i+1}-t_{i}\right)$

$\mathrm{C}$ is a constant that is independent of $\Delta$, and $\gamma$ is an integer or half-integer.

Definition 3 The Monte Carlo method is also referred as Random simulation method, Random sampling technique or Statistical testing method. Its basic idea is that in order to solve the problems of mathematics, physics, engineering technology and production management, firstly a probabilistic model or a random process should be established so that its parameters are equal to the solution of the problem. Then, the statistical characteristics of the parameters should be calculated by observing or sampling the model or process. Finally, the approximate value of the solution is given, and the accuracy of the solution is given. This can be expressed as a standard error of the estimate.

For the sake of simple discussion, this article assumes that financial markets are feasible and frictionless, that is, no taxes, no transaction fees, allowing short selling (borrowing securities and cash), and the deposit and loan interest rates of banks are the same. Assume again that the holder of this unconfirmed equity is a small investor and is self-financing, that is, the holder has neither added funds nor withdrew funds during the entire process. Here are some basic financial knowledge.

Definition 4 Arbitrage in financial markets is an important concept, that is, people are driven by interests and are always looking for opportunities for arbitrage. The intuitive meaning of arbitrage is that there is no capital at the beginning, after the capital's market operation, it becomes a non-negative (random) fund, and it has a positive probability of having a positive fund, which is commonly referred as "empty gloves and white wolf."

Definition 5 The market that satisfies the no-arbitrage hypothesis is called a viable market.

Definition 6 A European call option that targets a security (known as the variable of the target) refers to a contract between Party A (generally a securities company) and Party B at $0=t$. According to this contract, Party $\mathrm{B}$ has a right. A group of such securities can be purchased from Party A at time $T$ at a price $\mathrm{K}$ (called striking price or executable price), and if the market price $S_{T}$ at time $T$ is lower than $K$, Party B may not buy. According to this agreement, as long as the market price $S_{T}$ of the securities is higher than $K$ at time $T$, Party B will benefit. Therefore, Party B can get a net gain of $X_{T}=\left(S_{T}-K\right)^{+}$at time $T$. Among them, when $a^{+}=\left\{\begin{array}{ll}a & a>0 \\ 0 & a \leq 0\end{array}\right.$, this kind of contract is called option. The kind of option that only allows Party B to make a choice at final time $T$ is called European option. At this time, Party B wants $\mathrm{S}_{T}$ to be as large as possible in order to make more profits. That is, Party B, who has the right to choose, expects the stock to rise, so it is called a call option. In the opposite case, if $t=0$ Party A (usually the securities company) sells the following contract to Party B, this contract provides Party B with an authority to sell securities to Party A at the moment $T$ with price $K$. If the market price $S_{T}$ is higher than $K$ at time $T$, Party B may not sell. According to this contract, as long as the market price $S_{T}$ of the security is lower than $K$ at time $T$, the seller will benefit. Therefore, Party B can obtain a net gain of $X_{T}=\left(K-S_{T}\right)^{+}$at time $T$. Therefore, Party B expects the stock to fall, so it is called a put option.

For the sake of convenience, only European call options are studied and referred as European options for short in this paper.

Assuming that the operating law of an asset price follows the model (1), this chapter mainly studies the numerical simulation algorithm of profitability of the asset option, profit simulation error, and algorithm complexity. $T$ : the final moment, $K$ : execution price, P: Expected profit, ${ }_{p}^{-}$: the profit of numerical simulation, $\bar{S}(t)$ the price of the numerical simulation using Euler-Maruyama method at time t, second moment error is $\mathrm{E}\left(|P-\bar{P}|^{2}\right)$. The profit of European options studied in this chapter is in the following two forms.

$\mathrm{P}=S(T)-\inf _{0 \leq t \leq T} S(t), \mathrm{P}=\left(\sup _{0 \leq t \leq T} S(t)-K\right)^{+}$

The numerical simulation is in the form of

$\overline{\mathrm{p}}=\bar{S}(T)-\inf _{0 \leq t \leq T} \bar{S}(t), \overline{\mathrm{p}}=\left(\sup _{0 \leq t \leq T} \bar{S}(t)-K\right)^{+}$ 


\section{SIMULATING EUROPEAN OPTION PAYOFF BASED ON EULER-MARUYAMA AND MONTE CARLO ALGORITHM}

\subsection{Euler-Maruyama and Monte Carlo algorithms}

Lemma 1 If the $\operatorname{Eq}(1)$ satisfies the conditions of Assumptions 1 and 2, then there is a unique global positive solution on $\mathrm{t} \geq 0$ :

$$
\mathrm{ES}(t) \leq \xi(0) e^{\gamma t}, \forall t \geq 0 ; p \geq 1, E S^{p}(t) \leq \xi(0) e^{p\left[\gamma+0.5(p-1) K^{2}\right] t}, \forall t \geq 0
$$

and

$$
\mathrm{E}\left[\sup _{0 \leq t \leq T} S^{p}(t)\right] \leq \xi^{p}(0)\left(2+\frac{9 p^{2} K^{2}}{p\left[\gamma+0.5(p-1) K^{2}\right]}\right) e^{p\left[\gamma+0.5(p-1) K^{2}\right] T}, T \geq 0
$$

In this chapter, firstlt the Euler-Maruyama method is applied to discrete $\mathrm{Eq}(1)$ through the initial isometric step size $h$ to obtain the following discrete format:

$$
\begin{aligned}
& S_{n+1}=S_{n}\left[1+\gamma h+\mathrm{V}\left(S_{n-M}\right) \Delta W_{n}\right] \quad h=\frac{\tau}{M} \\
& \Delta W_{n}=((n+1) h)-W(n h), S_{n}=S\left(t_{n}\right)=S(n h) \\
& n=0,1,2, \ldots \ldots, m M-1 .
\end{aligned}
$$

Then, the numerical solution obtained from (2) is continuously constructed, thus constructing a continuous numerical solution orbit:

$$
\begin{gathered}
\bar{S}(t)=\xi(0)+\int_{0}^{t} \gamma x(u) \mathrm{d} u+\int{ }_{0}^{t} V(x(u-\tau)) x(u) \mathrm{d} W(u) \\
x(t)=\sum_{k=-M}^{m M-1} S_{k} I_{\left[t_{k}, t_{k+1}\right)}(t), t \geq 0, t_{k}=k h, \\
k=-M,-M+1, \cdots, 0,1, \cdots, m M-1 .
\end{gathered}
$$

It's easy to see

$\bar{S}(k h)=x(k h)=S\left(t_{k}\right), k=-M,-M+1, \cdots, 0,1, \cdots, m M-1$.

The mean value of the asset price at the final time $T$ is $E(S(T))$. The Euler-Maruyama discrete method (2) is used to obtain the sample orbit of the $\mathrm{N}$ asset price run, that is, the numerical solution sample orbit. Using the resulting $N$ sample orbits and applying the standard Monte Carlo method, we construct an estimate expression $\mu=\frac{1}{N} \sum_{i=1}^{N} S_{K}^{[i]}$ for the mean $E[S(T)]$, where $\mathrm{K}=\mathrm{mM}, S_{K}^{[i]}$ is an approximation of the asset price $S(T)$ at the final moment on the sample trajectory. The Euler-Maruyama and Monte Carlo algorithms are applied to simulate the global error of the asset price at the final moment as $E[S(T)]-\mu$.

The procedure for constructing the estimated expression $\mu$. by the standard Monte Carlo method is as follows:

For $i=1,2, \ldots, N$.

generate $\Delta W_{k}^{[i]} \quad k=1,2, \cdots \cdots, m M-1$

/ * $\Delta W_{k}^{[i]}=W_{k+1}^{[i]}-W_{k}^{[i]} \sim N(0, h) * /$

$S_{k+1}^{[i]}=S_{k}^{[i]}\left[1+\gamma h+V\left(S_{k-M}^{[i]}\right) \Delta W_{k}^{[i]}\right]$

$$
x(t)=\sum_{k=-M}^{m M-1} S_{k}^{[i]} I_{\left[t_{k}, t_{k+1}\right)}(t), t \geq 0
$$

where

$$
\begin{aligned}
& S_{S}^{-[i]}(T)=\xi(0)+\int_{0}^{T} \gamma x(u) \mathrm{d} u+\int_{0}^{T} V(x(u-\tau)) x(u) \mathrm{d} W^{[i]}(u) \\
& \backslash / * T: \text { final } \text { time }^{*} / \\
& \mu=\frac{1}{N} \sum_{i=1}^{N} S^{[i]}(T)
\end{aligned}
$$

/* $\bar{S}^{[i]}(\mathrm{T})$ : approximation to $S(T)$ at the $i$ sample $* /$

Theorem 1 If option Pricing Model Eq(1) satisfies Assumptions 1 and 2, applying the Euler-Maruyama method (2) and the standard Monte Carlo method (4) to simulate the profitability of this option, the global error of the asset price at the final moment $E[S(T)]-\mu$ the accuracy $O(\varepsilon)$ can be achieved. Then the value of the step length $h$ of the EulerMaruyama method and the number $N$ of the sample orbits of the standard Monte Carlo method can be determined by $\varepsilon$. Specially,

$h=\frac{\tau}{\left\lceil\frac{\tau}{\varepsilon}\right\rceil}, N=\left\lceil\frac{1}{\varepsilon^{2}}\right\rceil$

Proof: According to Minkowski inequality,

$P \geq 1, \mathrm{E}|\xi|^{p}<\infty, \mathrm{E}|\eta|^{p}<\infty$

$\left(\mathrm{E}|\xi+\eta|^{P}\right)^{1 / P} \leq\left(\mathrm{E}|\xi|^{P}\right)^{1 / P}+\left(\mathrm{E}|\eta|^{P}\right)^{1 / P}$

the Euler-Maruyama and Monte Carlo algorithms are used to analyse the global error of asset prices at the final moment as follows:

$$
\begin{aligned}
& \|\mathrm{E}[S(T)]-\mu\|_{2} \\
& =\left(\mathrm{E}[\mathrm{E}[S(T)]-\mu]^{2}\right)^{\frac{1}{2}} \\
& =\left(\mathrm{E}[\mathrm{E}[S(T)]-\mathrm{E}[\bar{S}(T)]+\mathrm{E}[\bar{S}(T)]-\mu]^{2}\right)^{\frac{1}{2}} \\
& \leq\left(\mathrm{E}[\mathrm{E}[S(T)]-\mathrm{E}[\bar{S}(T)]]^{2}\right)^{\frac{1}{2}}+\left(\mathrm{E}[\mathrm{E}[\bar{S}(T)]-\mu]^{2}\right)^{\frac{1}{2}}
\end{aligned}
$$

Because the Euler-Maruyama method has a weak convergence order of 1 order, there are

$\mathrm{E}[S(T)-\bar{S}(T)]=O(h), h \rightarrow 0$

The second term $E[\bar{S}(T)]-\mu$ on the right is the statistical error of the standard Monte Carlo experiment. From the perspective of the confidence interval

$\mathrm{E}[\bar{S}(T)]-\mu=O\left(\frac{1}{\sqrt{N}}\right), N \rightarrow \infty$

We then have

$\left(\mathrm{E}[\mathrm{E}[S(T)]-\mathrm{E}[\bar{S}(T)]]^{2}\right)^{\frac{1}{2}}+\left(\mathrm{E}[\mathrm{E}[\bar{S}(T)]-\mu]^{2}\right)^{\frac{1}{2}}$

$=O(h)+O\left(\frac{1}{\sqrt{N}}\right), h \rightarrow 0, N \rightarrow \infty$ 
$\|\mathrm{E}[S(T)]-\mu\|_{2}=O(\varepsilon), \varepsilon \rightarrow 0$

We thus get

$$
h=\frac{\tau}{\left\lceil\frac{\tau}{\varepsilon}\right\rceil}, N=\left\lceil\frac{1}{\varepsilon^{2}}\right\rceil
$$

The way that section titles and other headings are displayed in these instructions, is meant to be followed in your paper.

\subsection{Error analysis of option profit simulation}

This chapter assumes that the operating law of the asset price in the study follows $\mathrm{Eq}(1)$. It mainly studies the numerical simulation of European option profitability and the second-order moment error of profit numerical simulation. It mainly studies the profitability of the two options. The main difference between the two is that the final execution price is different. Taking the option profit $\mathrm{P}=\left(\mathrm{S}(\mathrm{T})-\inf _{0 \leq i \leq T} S(t)\right)$ as an example to illustrate the process of numerical modelling of option profit: First, the Euler-Maruyama method is used to numerically discretize the Model (2) to obtain a numerical sample orbit of the asset price over time. Then, the step size is determined by controlling the global error of the asset price at the final moment, and then the simulation $\bar{P}=(\bar{S}(\mathrm{~T})-$ $\left.{ }_{0 \leq i \leq T}^{i n f} \bar{S}(t)\right)$ is calculated. Finally, the second-order moment error of profit simulation is analysed.

Theorem 2 If option pricing Model (1) satisfies Assumptions 1 and 2, the Euler-Maruyama method (2) and the standard Monte Carlo method (4) are applied to simulate its option earnings, then the second moment error of the profit simulation is $O(h)$.

Proof: According to profitability and numerical simulation of European options,

$$
\mathrm{P}=S(T)-\inf _{0 \leq t \leq T} S(t), \overline{\mathrm{P}}=\bar{S}(T)-\inf _{0 \leq t \leq T} \bar{S}(t)
$$

the second-order moment error of this option profit simulation is

$$
\mathrm{E}\left(|\mathrm{P}-\overline{\mathrm{P}}|^{2}\right)=\mathrm{E}\left(\left|\left(S(T)-\inf _{0 \leq t \leq T} S(t)\right)-\left(\bar{S}(T)-\inf _{0 \leq t \leq T} \bar{S}(t)\right)\right|^{2}\right)
$$

Applying the formula again

$$
\begin{aligned}
& |a+b|^{2} \leq 2\left(|a|^{2}+|b|^{2}\right) \\
& \left|\inf _{0 \leq t \leq T} S(t)-\inf _{0 \leq t \leq T} \bar{S}(t)\right| \leq \sup _{0 \leq t \leq T}|S(t)-\bar{S}(t)|
\end{aligned}
$$

and the strong convergence order of the Euler-Maruyama method, a positive number $C$ can be found to satisfy

$$
\begin{aligned}
& \mathrm{E}\left(|\mathrm{P}-\overline{\mathrm{P}}|^{2}\right) \leq 2 \mathrm{E}\left(|S(T)-\bar{S}(T)|^{2}\right)+2 \mathrm{E}\left(\left|\inf _{0 \leq t \leq T} S(t)-\inf _{0 \leq t \leq T} \bar{S}(t)\right|^{2}\right) \\
& \leq 2 \mathrm{E}\left(|S(T)-\bar{S}(T)|^{2}\right)+2 \mathrm{E}\left(\sup _{0 \leq t \leq T}|S(t)-\bar{S}(t)|^{2}\right) \\
& \leq C h
\end{aligned}
$$

Similarly, we have
$\mathrm{P}=\left(\sup _{0 \leq t \leq T} S(t)-K\right)^{+}, \overline{\mathrm{P}}=\left(\sup _{0 \leq t \leq T} \bar{S}(t)-K\right)^{+}$

where $(\cdot)^{+} \equiv \max (\cdot, 0)$ The second-order moment error of this option profit simulation can be given as:

$$
\begin{aligned}
& \mathrm{E}\left(|\mathrm{P}-\overline{\mathrm{P}}|^{2}\right)=\mathrm{E}\left|\left(\sup _{0 \leq t \leq T} S(t)-K\right)^{+}-\left(\sup _{0 \leq t \leq T} \bar{S}(t)-K\right)^{+}\right|^{2} \\
& \leq \mathrm{E}\left|\sup _{0 \leq t \leq T} S(t)-\sup _{0 \leq t \leq T} \bar{S}(t)\right|^{2} \\
& \leq \mathrm{E}\left(\sup _{0 \leq t \leq T}|S(t)-\bar{S}(t)|^{2}\right) \\
& \leq C h
\end{aligned}
$$

\subsection{Numerical examples}

This section will support the main conclusions of this chapter through numerical examples.

Assume that the financial market is feasible and frictionless, that is, no taxes, no transaction fees, allowing short selling (borrowing securities and cash), and the deposit and loan interest rates of banks are the same. Assume again that the holder of this unconfirmed equity is a small investor and is self-financing, that is, the holder has neither added funds nor withdrew funds during the entire process.

The following autonomous random delay differential equation describes the operating law of an asset price:

$$
\left\{\begin{array}{l}
\mathrm{d} S(t)=0.05 S(t) \mathrm{d} t+0.2 S(t-0.5) \mathrm{d} W(t), \quad 0 \leq t \leq 1 \\
S(t)=1, \quad-0.5 \leq t \leq 0
\end{array}\right.
$$

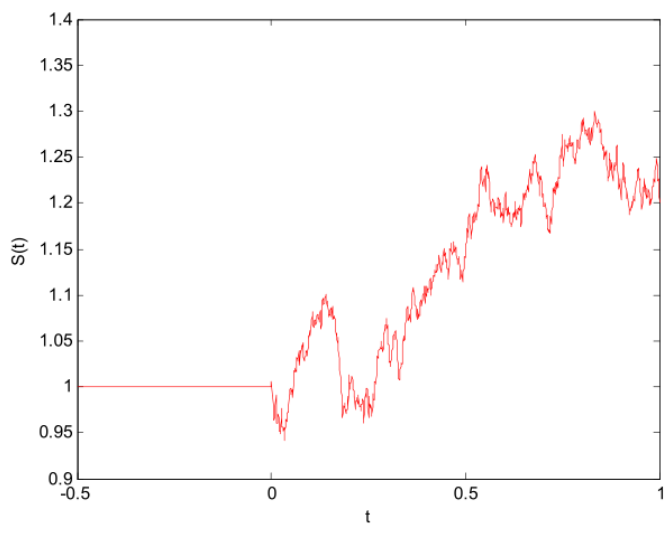

Figure 1. Price of asset with time

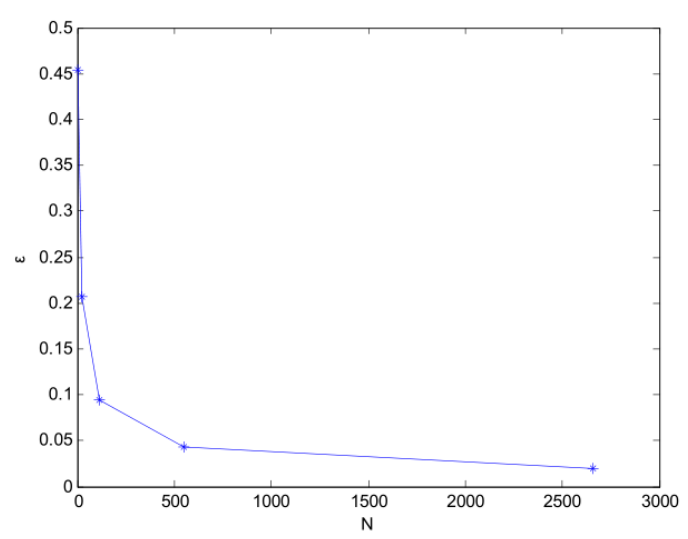

Figure 2. Numbers of sample path with overall error 
$S(t)$ denotes the market price of the asset at time $t$. At the final time $T=1$, the European-style call option profit of this asset at the last moment is $P=\max (0, S(1)-1)$, and the profit of the numerical simulation is $\bar{P}=\max (0, \bar{S}(1)-1)$, where $\bar{S}(1)$ represents the final moment of asset price simulation by using the numerical method.

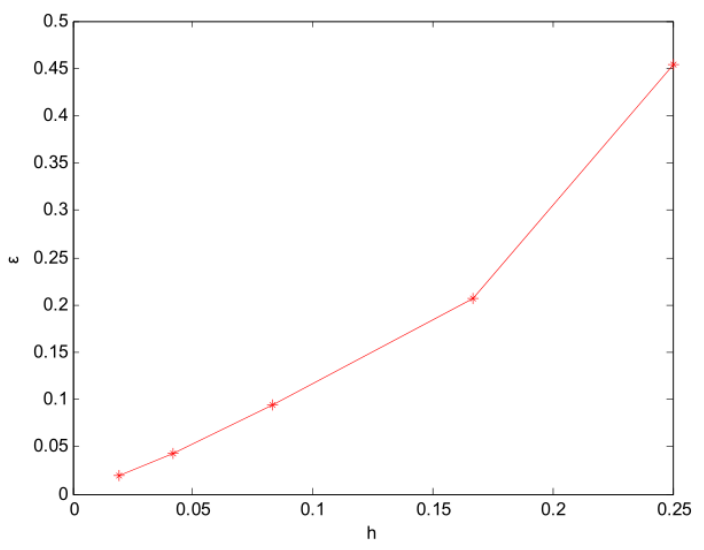

Figure 3. Step-size of Euler-Maruyama with overall error

The analytical solution of $\operatorname{Eq}(5)$ cannot be obtained directly, so its numerical solution is often used to approximate it. Here the Euler-Maruyama method of step size $h=\frac{1}{2^{10}}$ is used to discretize this equation and obtain an approximate representation of the analytical solution of Eq (5). Figure 1 can be obtained by applying the Matlab program. This chart depicts the changes of asset prices over time. The final asset price is $S(1)$

Euler-Maruyama method with a step size of $\frac{1}{2^{8}}$ is used to discretize this equation, and then the mean of 1000 sets of random sample data is used to represent $\mathrm{E}[S(1)]$, that is

$\omega_{i}: 1 \leq \mathrm{i} \leq 1000, E[S(1)]=\frac{1}{1000} \sum_{i=1}^{1000} S\left(1, \omega_{i}\right)$

\section{Euler-Maruyama}

method with $\mathrm{h}(\mathrm{j})=\frac{1}{2^{j}}, j=1,2,3,4,5$ and standard Monte Carlo method with the sample path number $\mathrm{N}(\mathrm{j})=4^{j}, j=$ $1,2,3,4,5$ are used to simulate the price of asset at final time $\bar{S}(1, j), j=1,2,3,4,5$ Then get the expression $\mu(\mathrm{j})=$ $\frac{1}{N(j)} \sum_{i=1}^{N(j)} \bar{S}(1, i), j=1,2,3,4,5 \quad$ The $\quad \bar{S}(1, i), j=1,2,3,4,5$ indicates that the asset price of the step size and sample size is $h(j), N(j), j=1,2,3,4,5$ at the final time $\mathrm{T}=1$. From the above calculation results, the global error expression $E[S(1)]-$ $\mu(j), j=1,2,3,4,5$ of the asset price at the final moment is expressed, and the Matlab program is used to obtain Figure 2 and Figure 3. The conclusion of Theorem 1 can be verified approximately by the figures, in which approximately. $h=$ $\frac{\tau}{\left\lceil\frac{\tau}{\varepsilon}\right\rceil}, N=\left\lceil\frac{1}{\varepsilon^{2}}\right\rceil$

\section{CONCLUSION}

For the option pricing Model (1), this chapter mainly studies the following two aspects.

Under certain conditions, the Euler-Maruyama method is applied to Equation (1) to obtain a sample orbit for the asset price run. The sample estimate $\mu=\frac{1}{N} \sum_{i=1}^{N} S_{K}^{[i]}$ of the asset price $E[S(T)]$ at the final moment is constructed by using the standard Monte Carlo method. If the global error $E[S(T)]-\mu$ of the asset price at the final moment can reach the precision $\mu=$ $\frac{1}{N} \sum_{i=1}^{N} S_{K}^{[i]}$, by controlling this global error, the step size $h=$ $\frac{\tau}{\left\lceil\frac{\tau}{\varepsilon}\right\rceil}$ and the sample number $N=\left\lceil\frac{1}{\varepsilon^{2}}\right\rceil$ can be deduced.

Based on the Euler-Maruyama method step size and the number of samples of the Monte Carlo method, the profitability of the option is simulated, and the second-order moment error of the profit simulation is derived.

\section{ACKNOWLEDGMENT}

This work was supported in part by the Philosophy and Social Science Research Program in Heilongjiang Province (No.16TJE01, 17TJD213).

\section{REFERENCES}

[1] Schonbucher PJ. (2003). Credit Derivatives Pricing Models: Models, Pricing and Implementation. John Wiley and Sons, Chichester, UK. 2-65.

[2] Mao XR. (1997). Stochastic Differential Equations and Their Applications. Harwood, New York. 5-86.

[3] Mao XR. (2006). Stochastic differential equations with Marconian switching. Imperial College, England. 1-76.

[4] Black F, Scholes M. (1973). The pricing of options and corporate liabilities. Journal of Political Economy, 81(3): 637-654.

[5] Hull J, White A. (1987). The Pricing of Options on Assets with Stochastic Volatilities. Journal of Finance, 42(2): 281-300.

[6] Merton RC. (1973). Theory of rational option pricing. Bell Journal of Economics and Management Science 4(1): 141183.

[7] Elliott RJ, Hoek JVD. (2003). A general fractional white noise theory and applications to finance. Mathematical Finance 13(2): 301-330.

[8] Guerra J, Nualart D. (2008). stochastic differential equations driven by fractional Brownian motion and standard Brownian motion. Stochastic Analysis and Applications 26(5): 1053-1075.

[9] Mishura YS. (2008). Stochastic calculus for fractional brownian motion and related processes. Springer-Verlag, Berlin, 5-60.

[10] Heston SI. (1993). A closed-form solution for options with stochastic volatility with applications to boud and currency options. Review of Financial Studies 6(2): 327-434.

[11] Liu XQ, Li CW. (2000). Weak approximation and extrapolations of stochastic differential equations with jumps. SIAM Journal on Numerical Analysis 37(6): 1747-1767.

[12] Bruti-Liberati N, Platen E. (2007). Approximation of jump diffusions in finance and economics. Computational Economics 29(3-4): 283-312.

[13] Mordecki E, Szepessy A, Tempone R, Zouraris GE. (2008). Adaptive weak approximation of diffusions with jumps. SIAM Journal on Numerical Analysis 46(4): 1732-1768.

[14] Mao XR, Rassias MJ. (2005). Khasminskii-type theorems for stochastic differential delay equations. Stochastic Analysis and Applications 23(5): 1045-1069.

[15] Wu F. (2008). Khasminskii-type theorems for neutral 
stochastic functional differential equations. Mathematica Applicata 21(4): 794-799.

[16] Mao XR. (2011). Numerical solutions of stochastic differential delay equations under the generalized Kaminski- type conditions. Applied Mathematics and Computation 217(12): 5512-5524. 